\title{
A Membrane-integrated Microfluidic Device to Study Permeation of Nanoparticles through Straight Micropores toward Rational Design of Nanomedicines
}

\author{
Naoki SASAKI, $* 1, * 2 \uparrow$ Mariko Tatanou, ${ }^{* 1}$ Tomoko SuZUKI, ${ }^{* 1}$ Yasutaka AnRaKU, $* 3$ \\ Akihiro KISHIMURA, ${ }^{* 3, * 4, * 5 \dagger}$ Kazunori KataOKA, ${ }^{* 3}$ and Kae SATO $* 1$ \\ *1 Department of Chemical and Biological Sciences, Faculty of Science, Japan Women's University, \\ 2-8-1 Mejirodai, Bunkyo, Tokyo 112-8681, Japan \\ *2 Department of Applied Chemistry, Faculty of Science and Engineering, Toyo University, 2100 Kujirai, \\ Kawagoe, Saitama 350-8585, Japan \\ *3 Department of Materials Engineering, Graduate School of Engineering, The University of Tokyo, \\ 7-3-1 Hongo, Bunkyo, Tokyo 113-8656, Japan \\ *4 Center for Molecular Systems, Kyushu University, 744 Moto-oka, Nishi, Fukuoka 819-0395, Japan \\ *5 Department of Applied Chemistry, Faculty of Engineering, Kyushu University, 744 Moto-oka, Nishi, \\ Fukuoka 819-0395, Japan
}

\begin{abstract}
Nanoparticles have been widely utilized to deliver drugs from blood vessels to target tissues. A crucial issue concerning nanoparticle-based drug delivery is to discuss the relationship between experimentally-obtained permeability and physical parameters. Although nanoparticles can permeate vascular pores, because the size and shape of the pores are essentially non-uniform, conventional animal testing and recent cell-based microfluidic devices are unable to precisely evaluate the effects of physical parameters (e.g. pore size and nanoparticle size) on permeation. In this study, we present a membraneintegrated microfluidic device to study permeation of nanoparticles through straight micropores. Porous membranes possessing uniform straight pores were utilized. The effects of pore size and pressure difference across the pores on nanoparticle permeation were examined. The experimentally determined permeability coefficient of $1.0 \mu \mathrm{m}$-pore membrane against $100 \mathrm{~nm}$-diameter nanoparticles agreed well with the theoretical value obtained for convectional permeation. Our method can be utilized to clarify the relationship between the experimentally-obtained permeability and physical parameters, and will help rational design of nanomedicines.
\end{abstract}

Keywords EPR effect, targeting, nanoDDS, nanomedicine, tumor, microchannel

(Received March 5, 2016; Accepted August 4, 2016; Published December 10, 2016)

\section{Introduction}

The ability to selectively deliver drugs to a target tissue is important for the efficient execution of drug medicinal effects. For example, if anticancer drugs are delivered selectively to a target tissue, the tissue will be efficiently treated and side effects will be reduced. The selective delivery of diagnostic agents, such as magnetic resonance imaging (MRI) contrast agents, luminescent probes and radioactive probes, is also useful in the clear imaging of a target tissue. To achieve such drug delivery, nanoparticles possessing a diameter of from several tens of nanometers to approximately $100 \mathrm{~nm}$ have been widely utilized. Various types of organic and inorganic nanoparticles have been tested, ${ }^{2}$ and the nanoparticle-based drug delivery system (nanoDDS) is a promising technique for next-generation therapies.

† To whom correspondence should be addressed.

E-mail: nsasaki@toyo.jp (N. S.); kishimura@mail.cstm.kyushu-u. ac.jp (A. K.)
The selective delivery of nanoparticles has been accounted for by the enhanced permeation and retention (EPR) effect. ${ }^{3,4}$ Briefly, although intravenously-administered nanoparticles are unable to permeate normal vascular walls, they can permeate tumor vascular walls that possess porous structures (Fig. 1(a)). Therefore, the increased permeability of tumor vascular walls against nanoparticles is thought to be strongly related to the pore size of vascular walls in addition to the size of the nanoparticles.

A number of studies have attempted to quantitatively clarify the origin of the increased permeability. For example, the pore size of tumor vascular walls has been estimated in several studies. Vick and Bigner reported that the size of gaps between endothelial cells in brain tumor microvasculature is 100 $250 \mathrm{~nm} .{ }^{5}$ Schlageter et al. reported that the shape of endothelial gaps in a brain tumor model is an ellipse, with a maximum minor axis of $0.38 \mu \mathrm{m}$ and a maximum major axis of $3.0 \mu \mathrm{m}{ }^{6}$ Hashizume et al. reported that the mean size of intercellular openings on tumor vessels in a mammary carcinoma is $1.7 \pm 0.1 \mu \mathrm{m}$, assuming the openings are circular. ${ }^{7}$ Since these values are one or two orders of magnitude greater than those of 
(a)

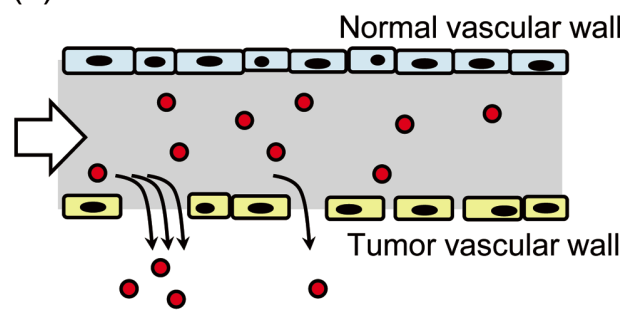

Nanoparticles $(\sim 100 \mathrm{~nm})$ (b)

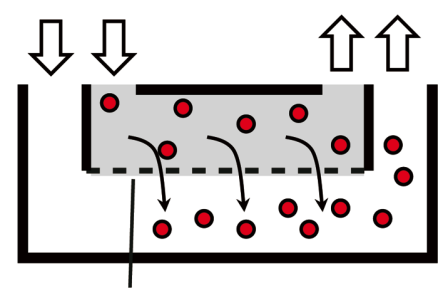

Porous membrane

Fig. 1 (a) Schematic illustration of nanoparticle permeation through a tumor vascular wall. (b) Schematic illustration of permeation test of nanoparticles through a porous membrane separating two microchannels.

normal endothelial gaps, it is clear that the pores of tumor vascular walls increase the permeability. Additionally, we reported that nanoparticles with sizes less than $150 \mathrm{~nm}$ can selectively accumulate in tumors ${ }^{8}$ suggesting that nanoparticle size is also important for increased permeability.

Most nanoDDS studies rely on animal testing, and although this approach is popular, it is unable to evaluate only the increased permeability of vascular walls because of the simultaneous lymphatic drainage of nanoparticles. Additionally, animal testing should be minimized since it is not without ethical concerns. Another testing system for nanoDDS is Transwell ${ }^{\mathrm{TM}}$, in which endothelial cells are cultured as a monolayer on a centimeter-sized porous membrane separating top and bottom chambers. The permeability of the cell monolayer can be determined by, for example, introducing fluorescently-labeled nanoparticles into the top chamber and measuring fluorescent intensity from the bottom chamber after a certain period of time. However, nanoparticles permeate the cell monolayer under static conditions, which differ from the dynamic conditions (e.g. shear stresses caused by blood flow) present in vivo. Also, the size of the system is larger than the microvessels found in tumors. Furthermore, in both testing systems - experimental animal and Transwell-, the size of intercellular pores is non-uniform and changes over time. Therefore, delineating the relationship between the experimentally-obtained permeability and physical parameters such as pore size, nanoparticle size, and so on, seems problematic. If the effects of the aforementioned physical parameters can be discussed individually, valuable information to improve nanoDDS by rational design of nanomedicines can be obtained.

In recent years, microfluidic devices have attracted great interest as a novel experimental model of microvessels. ${ }^{9,10}$ The size of a microfluidic channel can be adjusted to that of a microvessel, and various vascular cells can be cultured in the channel under blood flow-like flow conditions. ${ }^{11-13}$ In one approach, a porous membrane was integrated into a microfluidic device and utilized to evaluate the permeability of an endothelial monolayer on the membrane against fluorescein isothiocyanatelabeled bovine serum albumin (FITC-BSA) ${ }^{14-16}$ and lipid-coated nanoparticles. ${ }^{16}$ However, the pores within the monolayer are irregular in size and shape, as shown in previous studies. ${ }^{5-7}$ Therefore, from a different point of view, we have proposed an assay of nanoparticles utilizing the porous membrane with straight micropores with a defined pore size (without cells). ${ }^{17}$ The membrane has been utilized to evaluate convectional permeation of water under fluid flow, ${ }^{18}$ diffusional permeation of $\mathrm{CoFe}_{2} \mathrm{O}_{4}$ nanoparticles, ${ }^{19,20}$ but not to evaluate convectional permeation of nanoparticles for nanoDDS. The mechanism of nanoparticle permeation through vascular walls has not been clarified, ${ }^{21}$ and therefore convectional permeation of nanoparticles should be studied. Although convectional permeation of FITC-BSA has been investigated, ${ }^{14}$ the hydrodynamic diameter of FITC-BSA is around $9 \mathrm{~nm},{ }^{22}$ which is much smaller than the diameter of typical nanoparticles for nanoDDS. In addition, as described in the Results and Discussion section, we found that the theory in Ref. 14 is inappropriate for nanoparticles for nanoDDS. Therefore, if experimental results of convectional permeation of nanoparticles through straight micropores agreed with a theory of fluid dynamics, the relationship between the nanoparticle permeability and physical parameters is clarified for the first time, and these data will lead to rational design of nanomedicines.

In this study, we present an experimental model to study the permeation of nanoparticles through straight micropores under fluid flow. A porous membrane with straight micropores was integrated into a microfluidic device and utilized (Fig. 1(b)). Because the shape and size of the pores are uniform, the permeability of the membrane against nanoparticles can be precisely measured, and the relationship between permeability and nanoparticle size, pore size, and so on, can be determined. The results obtained are discussed together with a theory for the convectional permeation of hard spheres into pores. A membrane with non-uniform pore size and shape was also integrated into a microfluidic device to demonstrate the applicability of the model to non-uniform conditions.

\section{Experimental}

\section{Nanoparticles}

Block-aniomer poly(ethylene glycol)-poly $(\alpha, \beta$-aspartic acid) (PEG-PAsp; number-average molecular weight of PEG $=2000$, degree of polymerization (DP) of PAsp $=75$ ), homo-catiomer poly([5-aminopentyl]- $\alpha, \beta$-aspartamide) (Homo-P(Asp-AP); DP of $\mathrm{P}(\mathrm{Asp}-\mathrm{AP})=82)$, and Cy3-labelled PEG-PAsp were prepared as previously reported. ${ }^{23}$ 1-Ethyl-3-(3-dimethylaminopropyl) carbodiimide hydrochloride (EDC) was purchased from Wako Pure Chemical Industries (Osaka, Japan). Cy3 mono-reactive dye pack was purchased from GE Healthcare (Buckinghamshire, England).

In this study, size-tunable polyion complex vesicles (PICsome) were selected as a model nanoparticle. Cy3-labeled PICsome with a diameter of $101 \pm 3 \mathrm{~nm}$ and polydispersity index of $0.055 \pm 0.023$ was prepared as previously described. ${ }^{8,23}$ Briefly, PEG-PAsp, Cy3-labeled PEG-PAsp and Homo-P(Asp-AP) were 


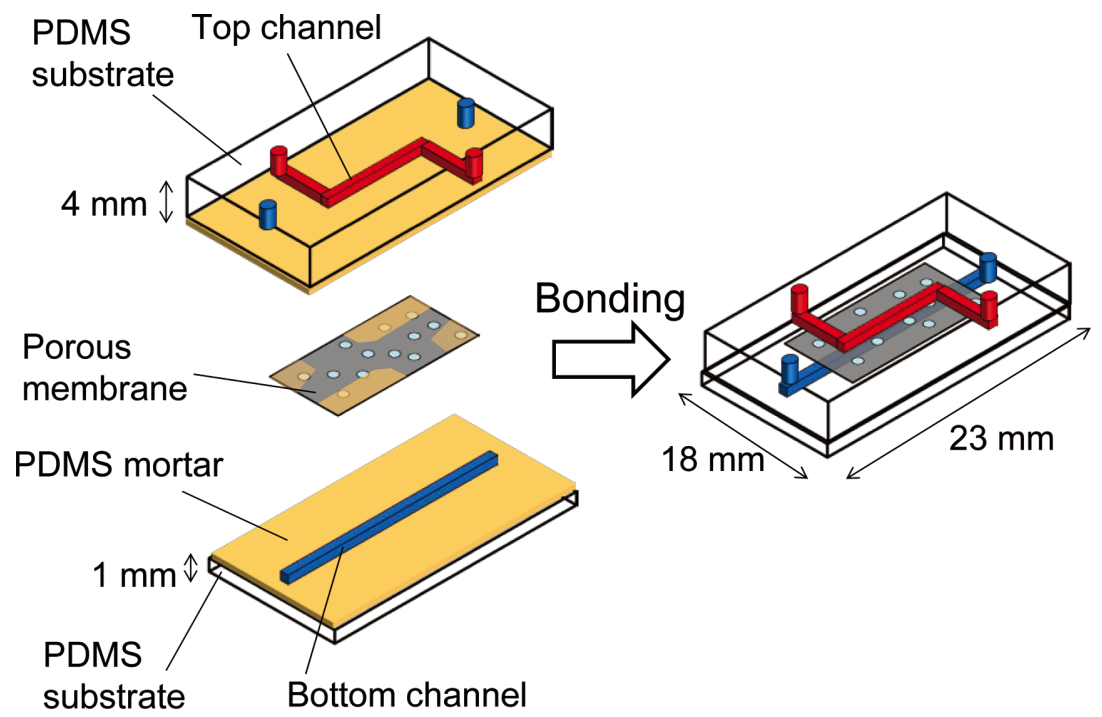

Fig. 2 Schematic illustration of fabrication of a membrane-integrated microfluidic device.

dissolved in $10 \mathrm{mM}$ phosphate buffer (PB, NaCl-free, $\mathrm{pH}$ 7.4) separately, with a polymer concentration of $1 \mathrm{mg} \mathrm{mL}^{-1}$. The solutions of PEG-PAsp and Cy3-labeled PEG-PAsp were blended in a volume ratio of 4:1. The resulting polyanion mixture was mixed at room temperature with the Homo-P(AspAP) solution in an equal unit ratio with respect to $-\mathrm{COO}^{-}$and $-\mathrm{NH}_{3}{ }^{+}$, followed by vortexing for 2 min to facilitate formation of the PICsomes. Then, a $10 \mathrm{mg} \mathrm{mL}^{-1}$ solution of EDC in PB was added to the PICsome solution for crosslinking. The reaction mixture was purified using a polyethersulfone ultrafiltration membrane (molecular weight cut-off; 300000). The size of the PICsomes was determined by dynamic light scattering using a Zetasizer Nano-ZS system (Malvern Instruments, Malvern, UK). Zeta potential of the PICsomes was confirmed using a Mobius (Wyatt Technology Corporation, Santa Barbara, CA, USA), and found to be almost zero in physiological salt concentrations (data not shown), so electrostatic effect on the PICsomes can be neglected.

\section{Porous membranes}

Commercially-available track-etched membranes ${ }^{24}$ were used in this study. Briefly speaking, the membranes are irradiated with ion beams to make latent tracks. Then, the membranes are etched chemically to form pores that are straight and uniform in size. Porous polyethylene terephthalate membranes with a pore size of 1.0 and $0.4 \mu \mathrm{m}$ were cut from cell culture inserts (353102 and 353493, Becton Dickinson and Company, Franklin Lakes, $\mathrm{NJ}$, USA) to a size of $3 \times 8 \mathrm{~mm}$. A porous polycarbonate membrane with a pore size of $0.1 \mu \mathrm{m}(110405$, Whatman, Kent, UK) was also prepared to the same size.

Porous membranes were characterized as follows. The thickness of the membranes was measured using a micrometer (MCD130-25, Niigata Seiki, Niigata, Japan). The size and density of the pores were measured using a scanning electron microscope (SEM; JSM-7001FA, JEOL Ltd., Tokyo, Japan) operating at $5.0 \mathrm{kV}$. Five SEM images were randomly acquired at different points on a membrane and utilized for further analysis. The cross sectional shape of the pores were characterized using another SEM (SU8220, Hitachi, Tokyo, Japan) operating at $7 \mathrm{kV}$.

\section{Device fabrication}

Microfluidic devices with porous membranes were fabricated as reported by Chueh et al. ${ }^{25}$ with some modification of the experimental procedures. A schematic illustration of the fabrication procedure is shown in Fig. 2. Poly(dimethylsiloxane) (PDMS, SILPOT 184, Dow Corning, Midland, MI, USA) was used to prepare substrates $(23 \times 18 \mathrm{~mm})$ with recessed microchannel patterns $(300 \mu \mathrm{m}$ width, $67 \mu \mathrm{m}$ depth, $10 \mathrm{~mm}$ length) as described elsewhere. ${ }^{12}$ A $1.0 \mu \mathrm{m}$-pore membrane was integrated into a microfluidic device as follows. First, a PDMS-hexane mixture with a weight ratio of 10 (prepolymer): 1 (curing agent):33 (hexane), referred to as PDMS mortar, was spin-coated on a glass slide at $2000 \mathrm{rpm}$ for $30 \mathrm{~s}$, and left for $10 \mathrm{~min}$ at room temperature to allow the hexane to evaporate. Then, the patterned PDMS substrates were stamped onto the glass slide and left for $2 \mathrm{~min}$. The substrates were peeled off, and the membrane was placed on a pattern of the top microchannel on a substrate. A small amount of PDMS mortar was placed on the edges of the membrane and left for $10 \mathrm{~min}$. Then, PDMS substrate with a bottom microchannel pattern was placed on the membrane. The PDMS-membrane composite was degassed for $30 \mathrm{~min}$, and then baked for $1 \mathrm{~h}$ at $100^{\circ} \mathrm{C}$ under pressure applied by a weight. For the $0.4 \mu \mathrm{m}$-pore membrane, PDMS mortar was spin-coated at $1000 \mathrm{rpm}$ on a glass slide, and the device was fabricated as described above. The $0.1 \mu \mathrm{m}$-pore membrane was also integrated as described above, except that PDMS mortar with a weight ratio of 10 (prepolymer): 1 (curing agent):60 (hexane) was placed on the edges of the membrane. The weight ratio (10:1:60) was determined by preliminary experiments to decrease the amount of uncured PDMS after hexane evaporation and to avoid channel clogging.

\section{Permeation tests}

Each end of the microchannels on a microfluidic device was connected to a silicone tube $(0.5 \mathrm{~mm}$ i.d., $1.0 \mathrm{~mm}$ o.d., $100 \mathrm{~mm}$ length, AS ONE, Osaka, Japan). The microfluidic device was degassed in a vacuum desiccator (PC-150K, Sanplatec, Osaka, Japan) connected to a vacuum pump (DAP-6D, Ulvac, Kanagawa, Japan) at $10 \mathrm{kPa}$ for $30 \mathrm{~min}$ to prevent air bubbles from remaining in the microchannel. ${ }^{26}$ The inlets of the top and bottom microchannels were connected to a $5-\mathrm{mL}$ syringe (1005TLL, Hamilton, Reno, NV, USA) and a $250-\mu \mathrm{L}$ syringe 

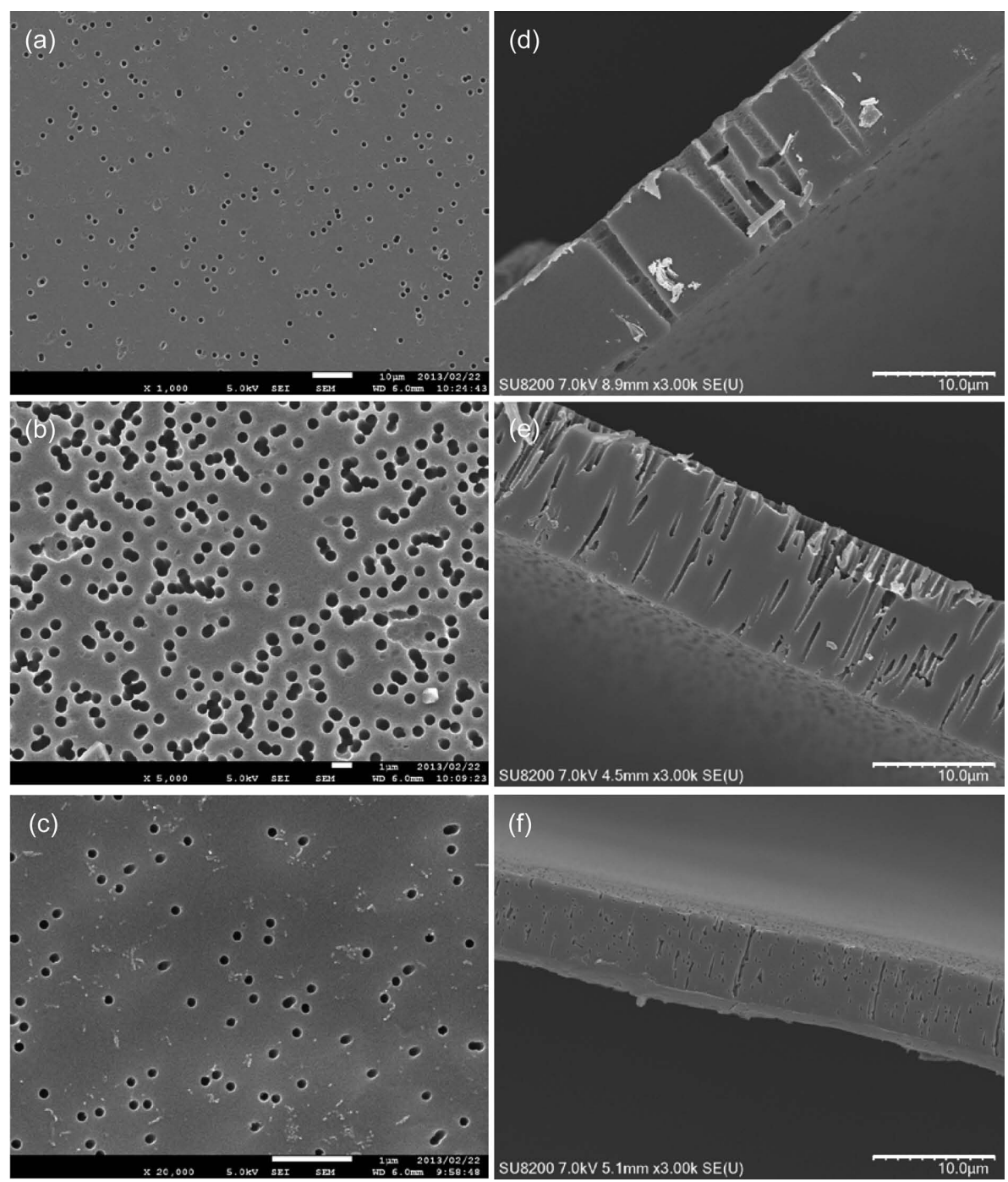

Fig. 3 SEM images of the porous membranes with a pore size of (a, d) 1.0, (b, e) 0.4, and (c, f) $0.1 \mu \mathrm{m}$. (a - c) Top view. (d-f) Cross view. Scale bar: (a) 10, (b, c) $1,(d-f) 10 \mu \mathrm{m}$.

(1725TLL, Hamilton), respectively, via a capillary. ${ }^{27}$

Permeation tests were conducted by introducing an aqueous test solution $\left(1 \mathrm{mg} \mathrm{mL}^{-1}\right.$ PICsome, $1 \times$ phosphate buffer saline (PBS, TAKARA BIO, Shiga, Japan), 0.5\% Tween 20 (SigmaAldrich, St. Louis, MO, USA)) into the top channel, and introducing another test solution $(1 \times$ PBS, $0.5 \%$ Tween 20$)$ into the bottom channel using syringe pumps (Model 210, KD Scientific, Holliston, MA, USA). The mean flow velocity at the inlet of the bottom channel $\left(v_{\mathrm{b} 0}\right)$ was maintained at $0.83 \mathrm{~mm} \mathrm{~s}^{-1}$, and the mean flow velocity at the inlet of the top channel $\left(v_{\mathrm{t} 0}\right)$ was changed in a stepwise fashion from 0.83 to $166 \mathrm{~mm} \mathrm{~s}^{-1}$. Bifurcation of the microchannels was observed to estimate the amount of permeated PICsome. The microchannels were observed using an inverted microscope (IX71, Olympus, Tokyo, Japan) equipped with a cooled CCD camera (Cascade 512F, Photometrics, Tucson, AZ, USA), 75-W xenon lamp, 10× objective lens (NA 0.30), and dichroic mirror block (86009, Chroma Technology, Bellow Falls, VT, USA, excitation $540-570 \mathrm{~nm}$ and emission 590-650 nm). Images were processed using image analysis software (Image J 1.45f, National Institutes of Health, MD, USA).

\section{Results and Discussion}

\section{Characterization of porous membranes}

Typical SEM images of the top view of the membranes are shown in Figs. 3(a) - 3(c). The observed pores were uniform in size for the $1.0 \mu \mathrm{m}$-pore membrane and $0.1 \mu \mathrm{m}$-pore membrane, while non-uniform for the $0.4 \mu \mathrm{m}$-pore membrane. Further analysis was conducted for the $1.0 \mu \mathrm{m}$-pore membrane and $0.4 \mu \mathrm{m}$-pore membrane. For the $1.0 \mu \mathrm{m}$-pore membrane, the radius of the pores $\left(r_{\mathrm{p}}\right)$ and the density of the pores $\left(N_{0}\right)$ were found to be $(5.60 \pm 0.47) \times 10^{-7} \mathrm{~m} \quad(N=970$ pores $)$ and $(1.77 \pm 0.10) \times 10^{10} \mathrm{~m}^{-2}(N=5$ images $)$, respectively. For the $0.4 \mu \mathrm{m}$-pore membrane, $r_{\mathrm{p}}$ and $N_{0}$ were found to be $(2.96 \pm 0.77) \times 10^{-7} \mathrm{~m}(N=1280$ pores $)$ and $(5.85 \pm 0.20) \times 10^{11}$ $\mathrm{m}^{-2}(N=5$ images), respectively. To demonstrate uniformity (or non-uniformity) of the membranes quantitatively, the ratio of the number of the non-uniform (connected) pores to that of all the pores was calculated from the SEM images, and found to be $4.3 \%$ (for the $1.0 \mu \mathrm{m}$-pore membrane) and $53.1 \%$ (for the $0.4 \mu \mathrm{m}$-pore membrane). Thickness of the membranes was $12 \mu \mathrm{m}$ for both membranes.

Typical SEM images of the cross view of the membranes are 
shown in Figs. 3(d) - 3(f). It was confirmed that the pores pierce the membranes. Some pores appeared to be disconnected in the images because they were not aligned in parallel to the cross section. In Fig. 3(d), cross section of the pore was imaged in a trapezoidal shape. This result suggests that if the pore is aligned in parallel to the cross section, the shape of the pore is not a true cylinder but a circular truncated cone. However, we did not find a considerable difference between the size of the openings on the one side of the membrane and that on the other side of the membrane (data not shown). This result means that the pores are true cylinders, not circular truncated cones.

\section{Fabrication of membrane-integrated microfluidic devices}

A photograph of a microfluidic device integrated with a $1.0 \mu \mathrm{m}$-pore membrane is shown in Fig. 4. Neither clearance between the PDMS substrates nor channel clogging was observed by microscopic visual inspection. Similar devices were successfully fabricated with a $0.4 \mu \mathrm{m}$-pore membrane and a $0.1 \mu \mathrm{m}$-pore membrane. The fabrication efficiency was $45 \%$ $(N=58)$ for the $1.0 \mu \mathrm{m}$-pore membrane, $56 \%(N=9)$ for the $0.4 \mu \mathrm{m}$-pore membrane, and $36 \%(N=11)$ for the $0.1 \mu \mathrm{m}$-pore membrane.

\section{Permeation tests}

For this study, PICsomes were selected as an example of a spherical nanoparticle. PICsomes have a tunable size ranging from $100-400 \mathrm{~nm}$ with narrow size distribution, and have been reported to show prolonged blood circulation and excellent tumor accumulation in mice for 100-nm PICsomes due to the

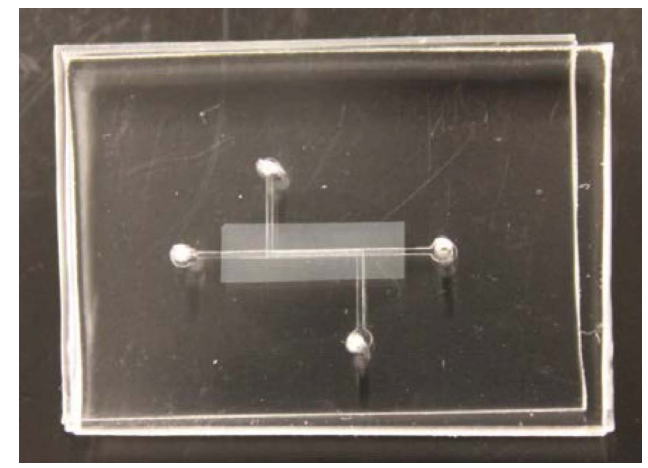

Fig. 4 A photograph of a membrane-integrated microfluidic device. Pore size: $1.0 \mu \mathrm{m}$.
EPR effect. ${ }^{8,23,28}$ Furthermore, 100-nm PICsomes can be used for MRI through encapsulation of superparamagnetic iron oxide nanoparticles, and have successfully been utilized in the detection of early-stage tumors. ${ }^{29}$

Figure 5 shows typical microscopic images of microfluidic channels in permeation tests. When $v_{\mathrm{t} 0}$ is equivalent to $v_{\mathrm{b} 0}$, virtually no fluorescence was observed from the bottom channel (Fig. 5(a)), which indicated negligible permeation of nanoparticles through the membrane pores under the experimental conditions. In contrast, fluorescence was observed from the bottom channel by increasing $v_{\mathrm{t} 0}$ (Fig. 5(b)), which indicated permeation of the nanoparticles by fluid flow through the pores. Permeation tests were conducted with membranes comprising different pore sizes, and the results are summarized in Fig. 6. C represents the normalized concentration of the nanoparticles, and is defined as the ratio of fluorescece intensity from the bottom channel after bifurcation to that from the top channel after bifurcation at $v_{\mathrm{t} 0}=166 \mathrm{~mm} \mathrm{~s}^{-1}$. At the $v_{\mathrm{t} 0}$, nanoparticle concentration in the top channel is almost the same as the original concentration. The $C$ value, which corresponds to the amount of permeated nanoparticles, increased with increasing $v_{\text {t0 }}$, increasing pore size, or both (Fig. 6(a)). Permeation was negligible when using the membrane with pore size of $0.1 \mu \mathrm{m}$, which is equivalent to the nanoparticle diameter. Therefore, results obtained with pore sizes of 1.0 and $0.4 \mu \mathrm{m}$ were further analyzed.

\section{Characterization of nanoparticle permeation based on a previous theory}

Firstly, we present a theoretical model on the permeation of nanoparticles through a porous membrane on a microfluidic device according to the report by Young et al. ${ }^{14}$ Schematic illustration of nanoparticle permeation through a porous membrane is shown in Figs. 7(a) and 7(b). The model is based on Darcy's law;

$$
v_{\mathrm{s}}=v_{\mathrm{p}} \phi=k \frac{\Delta P}{\mu \delta}
$$

where $v_{\mathrm{s}}\left(\mathrm{m} \mathrm{s}^{-1}\right), v_{\mathrm{p}}\left(\mathrm{m} \mathrm{s}^{-1}\right), \phi, k, \Delta P, \mu$, and $\delta$ represent the superficial (or apparent) velocity of the nanoparticles through the membrane, velocity of the nanoparticles inside the pores, porosity of the membrane, permeability coefficient, pressure difference across the membrane, viscosity of the solution (assumed to be $0.89 \mathrm{mPa} \cdot \mathrm{s}$ in this study), and thickness of the membrane, respectively.

A schematic illustration of the microfluidic device is shown in (a)

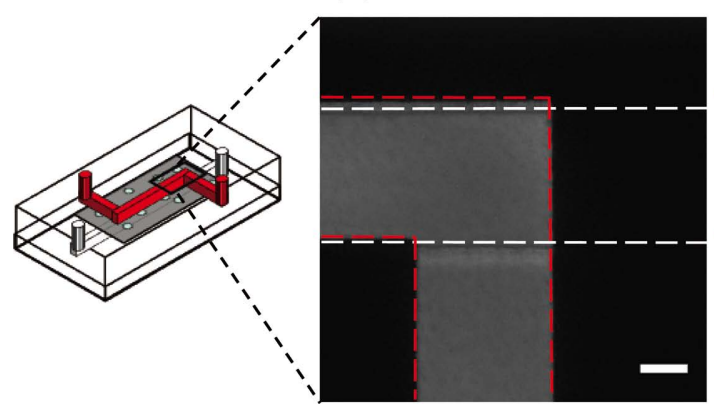

(b)

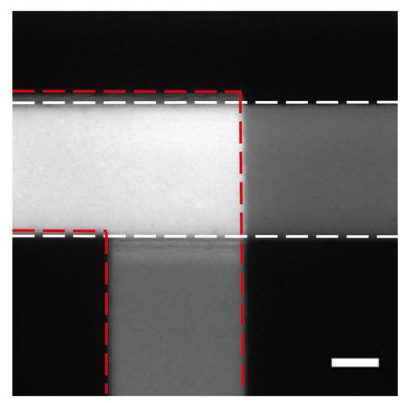

Fig. 5 Typical fluorescence micrographs of the permeation tests. Bifurcation of the microchannels is shown. Red: top channel. White: bottom channel. (a) $v_{\mathrm{t} 0}=0.83 \mathrm{~mm} \mathrm{~s}^{-1}$. (b) $v_{\mathrm{t} 0}=166 \mathrm{~mm} \mathrm{~s}^{-1}$. $v_{\mathrm{b} 0}$ was maintained at $0.83 \mathrm{~mm} \mathrm{~s}^{-1}$. Pore size: $1.0 \mu \mathrm{m}$. Scale bar: $100 \mu \mathrm{m}$. 

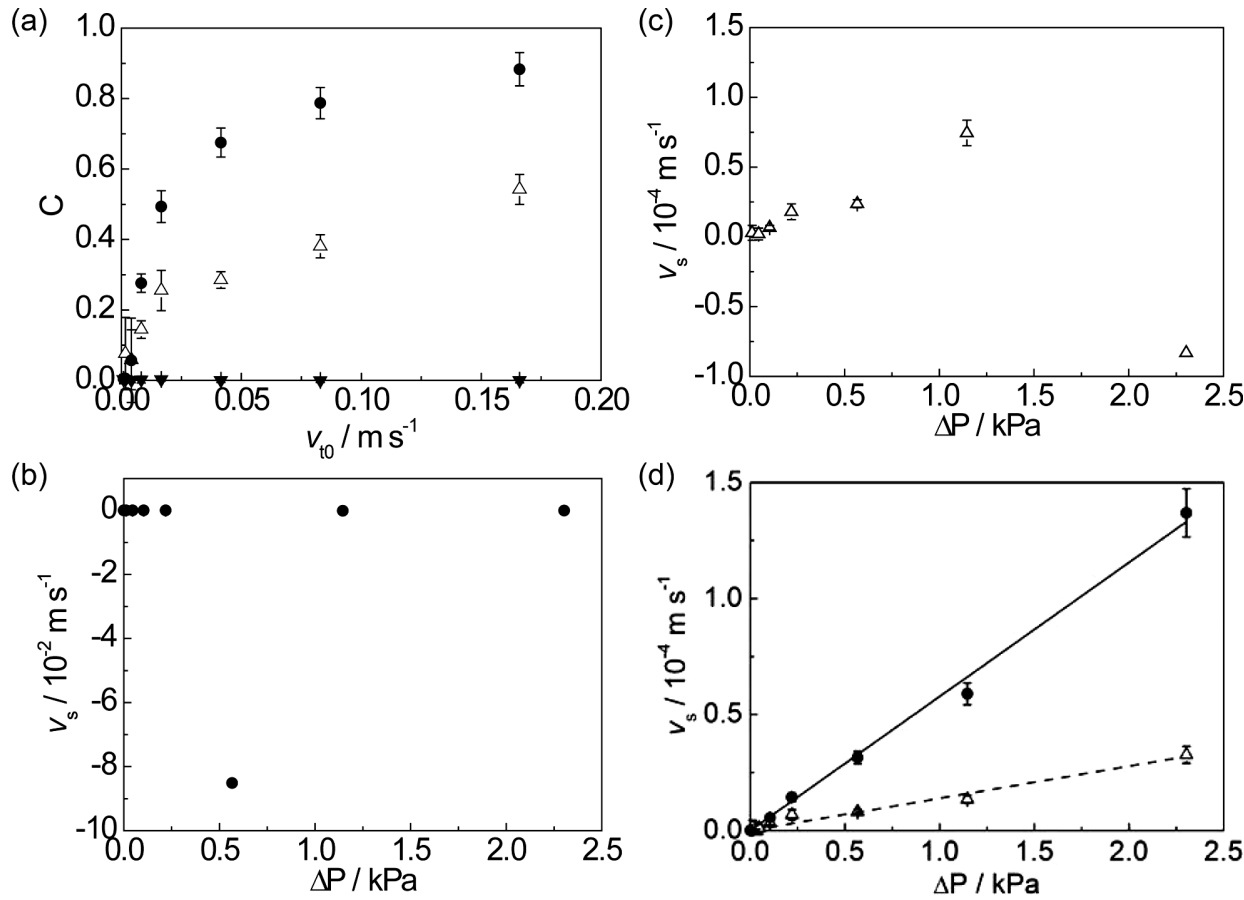

Fig. 6 (a) Dependence of $C$ on $v_{t 0}$. Pore size: $1.0 \mu \mathrm{m}$ (filled circles), $0.4 \mu \mathrm{m}$ (open triangles), and $0.1 \mu \mathrm{m}$ (filled inverted triangles). (b, c) Dependence of $v_{\mathrm{s}}$ on $\Delta P$. Pore size: (b) $1.0 \mu \mathrm{m}$, (c) $0.4 \mu \mathrm{m}$. The experimental results were analyzed by using a theoretical model in Ref. 14. $v_{\mathrm{s}}$ was positive for (b) $\Delta P<0.22 \mathrm{kPa}$ and (c) $\Delta P<1.15 \mathrm{kPa}$. However, further increase in $\Delta P$ resulted in negative $v_{\mathrm{s}}$ values, which indicates that the model used in Ref. 14 is not appropriate to evaluate the convectional permeation of nanoparticles through pores. (d) Dependence of $v_{\mathrm{s}}$ on $\Delta P$. Pore size: $1.0 \mu \mathrm{m}$ (filled circles), $0.4 \mu \mathrm{m}$ (open triangles). The experimental results were analyzed by using a theoretical model presented in this study. In Fig. 6(d), linear regression lines for pore sizes of $1.0 \mu \mathrm{m}$ (solid line) and $0.4 \mu \mathrm{m}$ (dashed line) are also shown. The error bars indicate $\pm 1 \mathrm{SD}$ of three experiments.

Figs. 7(c) and 7(d). The top and bottom channels are partly overlapped $\left(L_{1}, 4.6 \mathrm{~mm}\right.$ length) across the membrane, which allows nanoparticles to permeate from the top channel to the bottom channel. If the diffusion of the nanoparticles through the membrane is negligible compared to the convection of them, $v_{\mathrm{s}}$ can be described in a different form. Flux in the membrane pore $\left(J_{\mathrm{p}}\right)$ is described as;

$$
J_{\mathrm{p}}=f_{\mathrm{c}} v_{\mathrm{p}} C_{\mathrm{t} 0}
$$

where $f_{\mathrm{c}}$ represents the ratio of flux through porous media to that of flux in free solution, and will be discussed later. $C_{\mathrm{t} 0}$ represents the initial concentration of nanoparticles in the top channel. Flux in the bottom channel after bifurcation $\left(J_{\mathrm{f}}\right)$ is described as;

$$
J_{\mathrm{f}}=v_{\mathrm{b} 1} C_{\mathrm{f}}
$$

where $v_{\mathrm{b} 1}$ and $C_{\mathrm{f}}$ represent mean flow velocity and concentration of nanoparticles in the bottom channel after bifurcation, respectively. Considering the continuity of the fluids and the nanoparticles, the following equations are obtained;

$$
\begin{aligned}
& v_{\mathrm{b} 1} A_{\mathrm{b}}=v_{\mathrm{b} 0} A_{\mathrm{b}}+v_{\mathrm{s}} A_{\mathrm{m}} \\
& J_{\mathrm{f}} A_{\mathrm{b}}=J_{\mathrm{p}} A_{\mathrm{m}} \phi
\end{aligned}
$$

where $A_{\mathrm{b}}$ represents the area of cross section of the channel, $v_{\mathrm{b} 0}$ represents mean flow velocity at the inlet of the bottom channel, and $A_{\mathrm{m}}$ represents the area that two channels are overlapped. As mentioned in the Permeation tests section, $C$ is described as;

$$
C=\frac{C_{\mathrm{f}}}{C_{\mathrm{t} 0}}
$$

By using Eqs. (2) - (6), $C$ is described as;

$$
C=\frac{f_{\mathrm{c}} v_{\mathrm{s}} A_{\mathrm{m}}}{v_{\mathrm{b} 0} A_{\mathrm{b}}+v_{\mathrm{s}} A_{\mathrm{m}}}
$$

Using $A_{\mathrm{b}}=w h$ and $A_{\mathrm{m}}=w L_{2}$ (see Figs. 7(c) and 7(d)), $v_{\mathrm{s}}$ can be described as;

$$
v_{\mathrm{s}}=\frac{h v_{\mathrm{b} 0} C}{L_{1}\left(f_{\mathrm{c}}-C\right)}
$$

where $h$ represents the depth of the channel.

$f_{\mathrm{c}}$ represents the ratios of flux through porous media to that of flux in free solution. In the previous report by Young et al.,${ }^{14} f_{\mathrm{c}}$ is described as;

$$
f_{\mathrm{c}}=\left(1-\frac{s}{r_{\mathrm{p}}}\right)^{2}\left\{1-2.104 \frac{s}{r_{\mathrm{p}}}+2.09\left(\frac{s}{r_{\mathrm{p}}}\right)^{3}-0.95\left(\frac{s}{r_{\mathrm{p}}}\right)^{5}\right\}
$$

where $s$ represents the radius of the nanoparticles. The physical meaning of Eq. (9) has been explained by Friedman. ${ }^{30}$ Briefly, the first factor on the right side of Eq. (9) represents the steric 
(a)

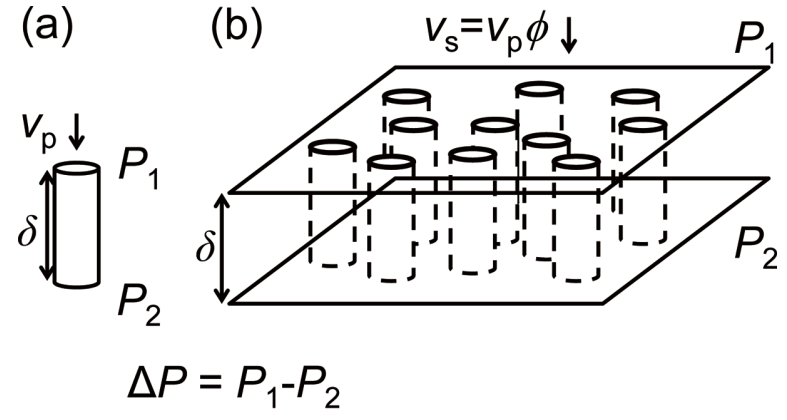

(c)

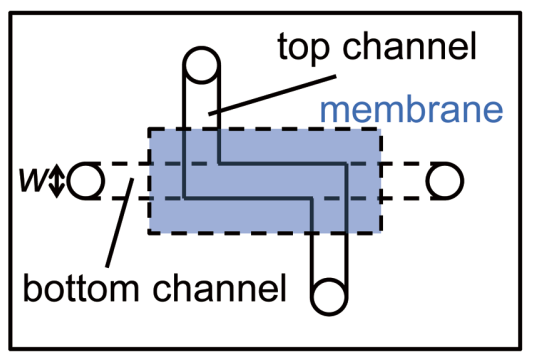

(d)

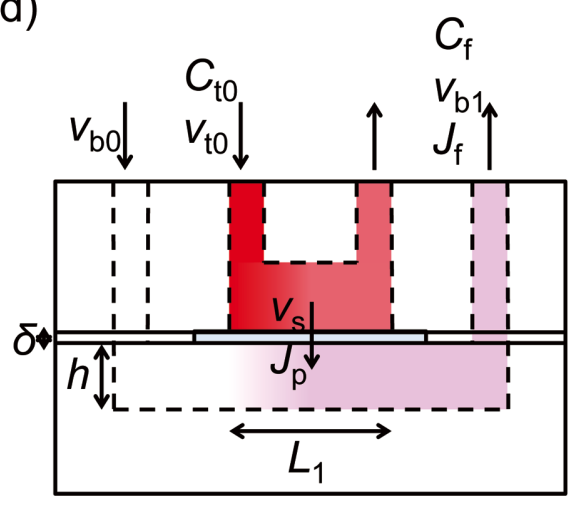

Fig. 7 (a, b) Schematic illustration of nanoparticle permeation through a porous membrane. (a) Permeation through a single pore. (b) Permeation through a porous membrane. (c, d) Schematic illustration of nanoparticle permeation on a membrane-integrated microfluidic device. (c) Top view. (d) Side view.

effect: that is, the nanoparticle entering the pore must be away at least a distance $s$ from the wall of the pore. The second factor on the right side of Eq. (9) represents the viscous effect: that is, once the nanoparticle enters the pore, because of the pore wall, it encounters a viscous resistance that exceeds the resistance in free solution. This factor is an approximation to the numerical solution of the governing flow equations. It should be pointed out that Eq. (9) describes solute diffusion, not convection.

$\Delta P$ was estimated according to the equation reported by Young et al. ${ }^{14}$ Briefly, mid-length pressures in the top and the bottom channel were considered. Velocity profiles in each channel were approximately obtained, and the pressure gradient along the microchannel was related to the second derivative of the velocity. Finally, $\Delta P$ was described as;

$$
\Delta P=6 \mu \frac{L_{2}}{h^{2}}\left(v_{\mathrm{t} 0}-v_{\mathrm{b} 0}\right)\left(\frac{m+1}{m}\right)
$$

where $L_{2}$ represents the total channel length. $m$ is an empirical constant to describe the effect of rectangular channel shape on the fluid flow; 31

$$
\begin{aligned}
& m=1.7+0.5 \alpha^{-1.4} \\
& \alpha=h / w
\end{aligned}
$$

Using $h$ of $67 \mu \mathrm{m}$ and $w$ of $300 \mu \mathrm{m}, m$ was calculated to be 5.78 .

Using equations shown above, $k$ can be estimated in the following manner. $\Delta P$ is estimated from Eq. (10) without any experimental results. $C$ is experimentally obtained as described in the Permeation tests section. Using the $C$ values, $v_{\mathrm{s}}$ can be estimated from Eq. (8). If a linear relationship is observed between $v_{\mathrm{s}}$ and $\Delta P$, Eq. (1) is valid, and the $k / \mu \delta$ value can be obtained from the slope of the regression line.

The dependence of $v_{\mathrm{s}}$ on $\Delta P$ for $1.0 \mu \mathrm{m}$ pore membrane and $0.4 \mu \mathrm{m}$ pore membrane is shown in Figs. 6(b) and 6(c), respectively. $v_{\mathrm{s}}$ was positive for $\Delta P<0.22 \mathrm{kPa}(1.0 \mu \mathrm{m}$ pore $)$ and $\Delta P<1.15 \mathrm{kPa}(0.4 \mu \mathrm{m}$ pore $)$. However, a further increase in $\Delta P$ resulted in negative $v_{\mathrm{s}}$ values, which indicates that the model used in the previous study ${ }^{14}$ is not appropriate to evaluate the convectional permeation of nanoparticles through pores. The reason for the discrepancy between the model and the experimental results seems to be the definition of $f_{\mathrm{c}}$.

Characterization of nanoparticle permeation based on the theory from this study

Next, we redefined $f_{\mathrm{c}}$ as an approximate equation of convectional permeation derived from numerical calculations; ${ }^{32}$

$$
f_{\mathrm{c}}=\left\{2\left(1-\frac{s}{r_{\mathrm{p}}}\right)^{2}-\left(1-\frac{s}{r_{\mathrm{p}}}\right)^{4}\right\}\left\{1-0.667\left(\frac{s}{r_{\mathrm{p}}}\right)^{2}-0.163\left(\frac{s}{r_{\mathrm{p}}}\right)^{3}\right\}
$$

The first and the second factor on the right side of Eq. (13) represent the steric and the viscous effect, respectively. By using Eq. (13), clearly, a linear relationship was observed between $v_{\mathrm{s}}$ and $\Delta P$ (Fig. 6(d)), which is consistent with Eq. (1). Therefore, permeation of the nanoparticles in the present study can be explained by the theoretical model presented in this study. The permeability coefficient was calculated to be $(6.62 \pm$ $0.39) \times 10^{-16} \mathrm{~m}^{2} \quad(1.0 \mu \mathrm{m}$ pore $)$ and $(1.03 \pm 0.11) \times 10^{-16} \mathrm{~m}^{2}$ $(0.4 \mu \mathrm{m}$ pore $)$ from the slopes of the regression lines.

\section{Comparison of permeability coefficients}

The theoretical permeability coefficient $\left(k_{0}\right)$ of the membrane was estimated using a cylindrical pore flow model ${ }^{14}$ with some modifications. Briefly, fluid flow in the pores is considered to be Hagen-Poiseuille flow, and the magnitude of the flow is considered to be reduced by a factor of $f_{\mathrm{c}}$. Therefore, $k_{0}$ can be obtained using:

$$
k_{0}=\frac{f_{\mathrm{c}} N_{0} \pi r_{\mathrm{p}}{ }^{4}}{8}
$$

For the $1.0 \mu \mathrm{m}$-pore membrane, using $s=5.0 \times 10^{-8} \mathrm{~m}, k_{0}$ was calculated to be $(6.62 \pm 1.18) \times 10^{-16} \mathrm{~m}^{2}$, which agreed well with the experimentally-obtained permeability coefficient $((6.62 \pm$ $\left.0.39) \times 10^{-16} \mathrm{~m}^{2}\right)$. Therefore, it was confirmed, for the first time, that the permeation of nanoparticles through straight micropores can be appropriately evaluated using the present theoretical model. For the $0.4 \mu \mathrm{m}$-pore membrane, using $s=5.0 \times 10^{-8} \mathrm{~m}$, $k_{0}$ was calculated to be $(1.56 \pm 0.81) \times 10^{-15} \mathrm{~m}^{2}$, which was larger than that of the experimentally-obtained permeability coefficient $\left((1.03 \pm 0.11) \times 10^{-16} \mathrm{~m}^{2}\right)$. This discrepancy between the theoretical and experimental results is natural since a large number of pores on the $0.4 \mu \mathrm{m}$-pore membrane are connected to 
each other as shown in Fig. 3(b). Future experiments utilizing membranes with different uniformity should clarify the nature of the discrepancy and, by comparing the permeability of uniform and non-uniform membranes, it should be possible to estimate the effect of non-uniformity on in vivo-permeability.

The present device is expected to be utilized to clarify the effects of various physical and chemical parameters on the nanoparticle permeation. For example, the charge of nanoparticles may affect the permeation via electrostatic interactions between the nanoparticles and the membranes. Adsorption of biomolecules on the nanoparticles may affect size, charge, and so on. Rigidity of the nanoparticles - hard or soft-may play a pivotal role because soft particles can be deformed to permeate into smaller pores. In addition to nanoparticle properties, membrane properties, e.g. zeta potential, elasticity, surface chemistry are matters of deep interest. We hope that the effects of these factors are tested and important parameters for the rational design of nanomedicines are clarified in future studies.

\section{Conclusions}

We have developed an experimental model to study the permeation of nanoparticles through straight micropores. The permeability coefficient of a $1.0 \mu \mathrm{m}$-pore membrane against nanoparticles was determined, and the permeability coefficients obtained agreed with the theoretically calculated value. The permeability coefficient was also determined using a non-uniform $0.4 \mu \mathrm{m}$-pore membrane, and demonstrated that the present model can be applied to the study of permeation through non-uniform pores. The present model can be utilized to clarify the relationship between the experimentally-obtained permeability and physical parameters, and will help rational design of nanomedicines.

\section{Acknowledgements}

This work was supported in part by the Tokyo Ohka Foundation for the Promotion of Science and Technology (to K. S.), Banyu Foundation Research Grant (to K. S.), Japan Society for the Promotion of Science (JSPS) KAKENHI (No. 23685037, 25107709 and 26288082 to A. K.; No. 25600065 to K. S.), JSPS through the Funding Program for World-Leading Innovative R\&D on Science and Technology (FIRST Program; to K. K.), and the Core Research for Evolutional Science and Technology (CREST) Program (to K. S.). SEM measurements were performed in part at the Center for Nano Lithography \& Analysis, The University of Tokyo, and supported by the Ministry of Education, Culture, Sports, Science and Technology (MEXT), Japan.

\section{References}

1. R. Duncan and R. Gaspar, Mol. Pharmaceutics, 2011, 8, 2101.

2. A. Z. Wilczewska, K. Niemirowicz, K. H. Markiewicz, and H. Car, Pharmacol. Rep., 2012, 64, 1020.

3. Y. Matsumura and H. Maeda, Cancer Res., 1986, 46, 6387.

4. H. Maeda, H. Nakamura, and J. Fang, Adv. Drug. Deliv.
Rev., 2013, 65, 71 .

5. N. A. Vick and D. D. Bigner, J. Neurolog. Sci., 1972, 17, 29.

6. K. E. Schlageter, P. Molnar, G. D. Lapin, and D. R. Groothuis, Microvasc. Res., 1999, 58, 312.

7. H. Hashizume, P. Baluk, S. Morikawa, J. W. McLean, G. Thurston, S. Roberge, R. K. Jain, and D. M. McDonald, Am. J. Pathol., 2000, 156, 1363.

8. Y. Anraku, A. Kishimura, A. Kobayashi, M. Oba, and K. Kataoka, Chem. Commun., 2011, 47, 6054.

9. K. Sato, N. Sasaki, H. A. Svahn, and K. Sato, Adv. Drug. Deliv. Rev., 2014, 74, 115.

10. N. Sasaki, J.-i. Jo, I. Aoki, and K. Sato, Anal. Biochem., 2014, 458, 72 .

11. Y. Tanaka, Y. Kikukawa, K. Sato, Y. Sugii, and T. Kitamori, Anal. Sci., 2007, 23, 261.

12. N. Sasaki, M. Shinjo, S. Hirakawa, M. Nishinaka, Y. Tanaka, K. Mawatari, T. Kitamori, and K. Sato, Electrophoresis, 2012, 33, 1729.

13. M. Sato, N. Sasaki, M. Ato, S. Hirakawa, K. Sato, and K. Sato, PLoS ONE, 2015, 10, e0137301.

14. E. W. K. Young, M. W. L. Watson, S. Srigunapalan, A. R. Wheeler, and C. A. Simmons, Anal. Chem., 2010, 82, 808.

15. J. Shao, L. Wu, J. Wu, Y. Zheng, H. Zhao, X. Lou, Q. Jin, and J. Zhao, Biomed. Microdev., 2010, 12, 81.

16. Y. Kim, M. E. Lobatto, T. Kawahara, B. L. Chung, A. J. Mieszawska, B. L. Sanchez-Gaytan, F. Fay, M. L. Senders, C. Calcagno, J. Becraft, M. Tun Saung, R. E. Gordon, E. S. G. Stroes, M. Ma, O. C. Farokhzad, Z. A. Fayad, W. J. M. Mulder, and R. Langer, Proc. Natl. Acad. Sci. U. S. A., 2014, 111, 1078 .

17. N. Sasaki, M. Tatanou, Y. Anraku, A. Kishimura, K Kataoka, and K. Sato, Proceedings of Micro Total Analysis Systems 2013, 2013, 1818.

18. N. Miyaoi, H. Ohashi, T. Ito, and T. Yamaguchi, J. Chem. Eng. Jpn., 2008, 41, 766.

19. J. R. Stephens, J. S. Beveridge, A. H. Latham, and M. E. Williams, Anal. Chem., 2010, 82, 3155.

20. J. R. Stephens, J. S. Beveridge, and M. E. Williams, Analyst, 2011, 136, 3797.

21. M. R. Kano, Adv. Drug. Deliv. Rev., 2014, 74, 2.

22. U. Böhme and U. Scheler, Chem. Phys. Lett., 2007, 435, 342.

23. Y. Anraku, A. Kishimura, M. Oba, Y. Yamasaki, and K. Kataoka, J. Am. Chem. Soc., 2010, 132, 1631.

24. P. Apel, Radiat. Meas., 2001, 34, 559.

25. B.-h. Chueh, D. Huh, C. R. Kyrtsos, T. Houssin, N. Futai, and S. Takayama, Anal. Chem., 2007, 79, 3504.

26. K. Hosokawa, K. Sato, N. Ichikawa, and M. Maeda, Lab Chip, 2004, 4, 181.

27. N. Sasaki, A. Takemura, and K. Sato, Electrophoresis, 2012, 33, 3159.

28. A. Kishimura, Polymer J., 2013, 45, 892.

29. D. Kokuryo, Y. Anraku, A. Kishimura, S. Tanaka, M. R. Kano, J. Kershaw, N. Nishiyama, T. Saga, I. Aoki, and K. Kataoka, J. Control. Release, 2013, 169, 220.

30. M. H. Friedman, "Principles and Models of Biological Transport", 2008, Springer, USA.

31. N. M. Natarajan and Lakshmanan, Indian J. Technol., 1972, 10,435

32. J. L. Anderson and J. A. Quinn, Biophys. J., 1974, 14, 130. 